\title{
Methylene Blue Does Not Have to Be Considered Only as Rescue Therapy for Distributive Shock
}

\author{
Paulo Roberto Barbosa Evora
}

Published online: 29 September 2013

(C) American College of Medical Toxicology 2013

\section{Dear Editor}

Jang and colleagues [1] published a recent review considering methylene blue (MB) as a "potential new use of an old antidote" to treat distributive shock. The review included three concise concluding remarks. (1) Increased nitric oxide (NO), and cyclic guanosine monophosphate (cGMP) production is well documented in various conditions of distributive shock such as sepsis and anaphylaxis; (2) The NO-cGMP pathway plays a central pathophysiology role; and (3) There are experimental and clinical evidences that support MB use in cases of refractory vasodilatory shock with no response to conventional treatment. This third conclusion, considering MB as a last rescue therapeutic option, deserves some consideration.

In 2009, targeting MB for distributive shock treatment in heart surgery, we published a personal report including 15 years (now 19 years) of questions, answers, doubts, and certainties [2]. I have reported, over and over, some pivotal observations: (1) In the recommended doses, MB is safe (the lethal dose is $40 \mathrm{mg} /$ $\mathrm{kg}$ ); (2) MB did not cause endothelial dysfunction; (3) The MB effect appears in cases of $\mathrm{NO}$ upregulation; and (4) MB is not a vasoconstrictor, by blocking the cGMP pathway, it releases the cAMP pathway, facilitating the norepinephrine vasoconstrictor effect; (5) The most used dosage is $2 \mathrm{mg} / \mathrm{kg}$ as IV bolus followed by the same continuous infusion because plasma concentrations strongly decays in the first $40 \mathrm{~min}$; and (6) There is a possible "window of opportunity" for the MB's effectiveness [2].

Blacker and Whalen reported a distributive shock case during an on-pump coronary artery bypass grafting with no response to MB [3]. A possible explanation was given based on Fernandes and colleagues' academic thesis using a mouse sepsis model that evidenced three 8-h windows of guanylate cyclase (GC) activity [4]. In the first $8 \mathrm{~h}$, there is increased nitric oxide synthase (NOS)

P. R. B. Evora $(\bowtie)$

Ribeirão Peto School of Medicine, University of São Paulo, Ribeirão

Preto, São Paulo, Brazil

e-mail: prbevora@gmail.com activity and GC upregulation. In the second $8 \mathrm{~h}$, there is an absence of GC expression and a downregulation of NOS. In the third 8-h window, there is an upregulation of GC and NOS. The authors emphasized two practical and education fundamental aspects: (1) The disclosure in using the MB treatment considering the window opportunity and (2) The need for the establishment of this window in humans, perhaps choosing cGMP as biomarker since our attempt to use nitrite/nitrate, measured by chemiluminescence, was frustrating [5]. I wrote another quite similar letter (still under review) with congratulations to Blacker and Whalen by the attitude of seeking, in retrospect, why MB had only a transient effect and not just dismissing its action as often happens. In addition, I tried to introduce an easier concept to understand the "Window of Opportunity" definition... "MB action to treat distributive shock is time-dependent."

In conclusion, $\mathrm{MB}$ use as a last rescue therapeutic option is against the above mentioned concepts, and it is possible that $\mathrm{MB}$ does not act (second window), or acts too late (third window) when the circulatory shock is metabolically irreversible presenting high lactate levels and intractable metabolic acidosis. Maybe it is more sensible to consider MB not as a late rescue treatment, but as an adjuvant drug to be used precociously (window 1).

\section{References}

1. Jang DH, Nelson LS, Hoffman RS (2013) Methylene blue for distributive shock: a potential new use of an old antidote $\mathrm{j}$ med toxicol. In press.

2. Evora PR, Ribeiro PJ, Vicente WV et al (2009) Methylene blue for vasoplegic syndrome treatment in heart surgery: fifteen years of questions, answers, doubts and certainties. Rev Bras Cir Cardiovasc 24(3):279-288

3. Blacker SN, Whalen FX (2013) Vasoplegic syndrome: does the timing of methylene blue matter? J Anesthe Clinic Res 4:333

4. Fernandes D, da Silva-Santos JE, Duma D et al (2006) Nitric oxidedependent reduction in soluble guanylate cyclase functionality accounts for early lipopolysaccharide-induced changes in vascular reactivity. Mol Pharmacol 69(3):983-990

5. Viaro F, Baldo CF, Capellini VK et al (2008) Plasma nitrate/nitrite (NOx) is not a useful biomarker to predict inherent cardiopulmonary bypass inflammatory response. J Card Surg 23(4):336-338 